\title{
Role of Leishmanin Skin Test (LST) as Epidemiological Indicator for Cutaneous Leishmaniasis in Al-tragma Village, River Nile State, Sudan
}

\author{
Abdelgani Mohamed Osman' ${ }^{1}$, Adam Dawoud Abakar ${ }^{\circledR}{ }^{\circledR}$, Nazar Mohamed Abdalla ${ }^{3}$, Kamal Hussain', \\ Rasha Slaha El-deen Hassan ${ }^{4}$, Khalid Abdelsamea Mohamedahmed ${ }^{5,6^{*}}{ }^{\infty}$, Saseed Bakiker Mahmoud ${ }^{7}$ \\ 'Department of Parasitology, Faculty of Medicine and Health Sciences, University of Technology Merowe, Merowe, \\ Sudan \\ ${ }^{2}$ Department of Medical Parasitology, Faculty of Medical Laboratory Sciences, Gezira University, Wad Medani, \\ Sudan \\ ${ }^{3}$ Department of Internal Medicine, Faculty of Medicine, Abaha University, Abaha, KSA \\ ${ }^{4}$ Department of Clinical Pharmacy, Faculty of Pharmacy, University of Khartoum, Khartoum, Sudan \\ ${ }^{5}$ Department of Hematology, Faculty of Medical Laboratory Sciences, Gezira University, Wad Medani, Sudan \\ ${ }^{6}$ Department of Immunology, Faculty of Medical Laboratory Sciences, Gezira University, Wad Medani, Sudan \\ ${ }^{7}$ Department of Dermatology and Venereal Diseases, Faculty of Medicine, Gezira University, Wad Medani, Sudan
}

\begin{abstract}
Introduction: Cutaneous leishmaniasis $(\mathrm{CL})$ is a common health problem in the world and its zoonotic form has been reported to occur in Sudan. Leishmaniasis has been considered the second most important parasitic disease after malaria. Therefore, the role of leishmanin skin test (LST) as an epidemiological indicator for CL infection was determined in Al-tragma village, one of the endemic areas for the disease in Sudan.

Materials and Methods: In this cross-sectional study, 410 individuals inhabiting Al-tragma village were enrolled. Two methods of data collection were used in this study, a structured questionnaire and LST. A structured questionnaire was designed to collect and test socio-demographic characteristics. The LST was performed by intradermal injection of $0.1 \mathrm{~mL}$ of LST antigen on the volar surface of the left forearm. The result of LST was read after 48-72 hours using the ballpoint pen technique. SPSS version 20.0 was used for data analysis.

Results: LST results showed that $70.7 \%$ of respondents were positive and $29.3 \%$ were negative. The most infected age groups were $31-40$ years $(80 \%)$ and more than 40 years $(82 \%)$. The results showed there was a significant difference in LTS results based on age groups, occupations, domestic animals and pets found in and around the houses, and previous experience of the disease $(P<0.05)$.

Conclusion: The overall positive rate for LST was $70.7 \%$. The exposure duration had no effect on LST results. It seems that the patients acquired long-lasting immunity. LST is still a promising tool for evaluating the epidemiological status of Leishmaniasis in endemic areas.

Keywords: Leishmanin skin test, Leishmaniasis, Cutaneous leishmaniasis, Leishmania major, Sudan
\end{abstract}

Received: September 10, 2020, Accepted: December 9, 2020, ePublished: January 1, 2021

\section{Introduction}

Leishmaniasis is one of the neglected tropical diseases and vector-borne diseases, present in different clinical forms $(1,2)$. Leishmaniasis comprises a group of diseases caused by intracellular kinetoplastid protozoan parasites of the genus Leishmania or Viannia that live in macrophages of some vertebrates as amastigote form (3).

The Leishmania parasites consist of different species and strains with epidemiological diversity that are pathogenic to humans, leading to different pathological and clinical diseases such as visceral leishmaniasis, mucocutaneous leishmaniasis (MCL), diffuse-cutaneous leishmaniasis (DCL), and cutaneous leishmaniasis (CL), in which the parasite is restricted to cutaneous and subcutaneous tissue (4).

$\mathrm{CL}$ is a common disease in the world, which is divided into zoonotic cutaneous leishmaniasis (ZCL) and anthroponotic cutaneous leishmaniasis (ACL) (5). ZCL was reported in Sudan and has been considered to be the second most important parasitic disease after malaria. According to the reports of the World Health Organization (WHO), many cases have been reported in 
Khartoum State (6), and there was an increased incidence of the disease from October to last March 2014, and about 560 patients have been reported to be infected, most of whom were from East Nile (Shrug Al-Nile), Salha and Al-Klaklah (personal communication). Another survey conducted in Sudan showed that of the 1236 individuals enrolled in this survey, 688 were diagnosed with CL, with an infection rate of $55.7 \%$ (6). Leishmaniasis is prevalent in the 4 continents and it is considered endemic in 88 countries, 72 of which are developing countries. Worldwide, the annual prevalence of new cases is estimated to be $0.2-0.4$ million for visceral leishmaniasis and 0.71.2 million for CL, while 12 million people are currently affected by the disease (7). The diagnosis of CL depends on clinical examination coupled with parasitological and molecular techniques. The parasitological investigations, which are considered as a gold standard technique, face many problems, including low parasite count in the lesion and the presence of the parasite restricted to skin only. Traditional methods, such as direct culture, are time-consuming and can be performed in specialized laboratories by expert microscopists, while molecular methods have many advantages, including high sensitivity and specificity (8).

The current study aimed to assess the role of leishmanin skin test (LST) as an epidemiological indicator for CL in the study area.

\section{Materials and Methods \\ Study Area}

This study was conducted in El Tragma Elgabha Village. It is located at the eastern bank of the River Nile (16.4 o North and $33.25^{\circ}$ East), $13 \mathrm{Km}$ north of Shandi city, River Nile State, and $175 \mathrm{~km}$ north of Khartoum, the capital of Sudan (Figure 1). The population is around 5000 individuals. The area is endemic for CL where an outbreak of CL was reported in 1978. It was agricultural in nature that provided a good environment for survival and multiplication of insect vectors (9).

\section{Experimental Design}

This cross-sectional study was conducted to describe the epidemiological status of CL using the LST and a questionnaire.

\section{Questionnaire Design}

A structured questionnaire was designed and used to collect socio-demographic characteristics. Data concerning exposure, background of inhabitants, their past complaints, and accompanied skin lesions were also included.

\section{Sample Size}

The sample size was determined in a systematic way according to the following simple formula of Daniel ( $\mathrm{n}=$ $\left.\mathrm{Z}^{2} \mathrm{P}(1-\mathrm{P}) / \mathrm{d}^{2}\right)$, where $\mathrm{n}$ is the sample size, $\mathrm{Z}$ is the $\mathrm{Z}$ statistic for the level of confidence, $P$ is expected prevalence or proportion (in proportion of one; if $20 \%, P=0.2$ ), and $\mathrm{d}$ is the precision (in proportion of one; if $5 \%, d=0.05$ ). $Z$ statistic (Z): For the level of confidence of $95 \%$ which is conventional, $\mathrm{Z}$ value is 1.96 (10).

\section{Leishmanin Skin Test}

Leishmanin is a reagent recommended for skin tests in humans for Immuno-epidemiological studies (11).

The Leishmanin antigen obtained from Pasteur Institute of Iran and L. major (reference strain MRHO/IR/75/ ER) was used for the preparation of the leishmanin. The preparation contained a final concentration of $L$. major promastigotes of $6 \times 10^{6}$ in $1 \mathrm{~mL}$ of phosphate-buffered saline.

The LST was performed by intradermal injection of $0.1 \mathrm{~mL}$ of skin test antigen on the volar surface of the left forearm using a sterile disposable insulin syringe. The results of LST were read after 48-72 hours using the ballpoint pen technique; induration size of $\geq 5 \mathrm{~mm}$

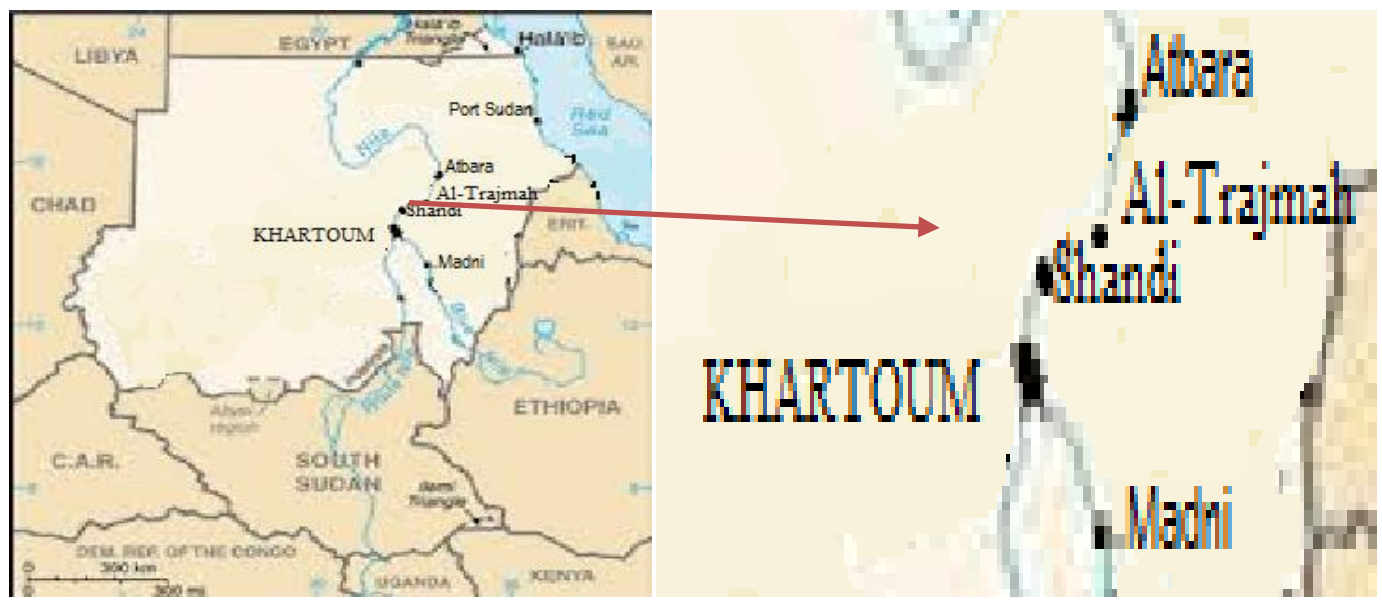

Figure 1. Location of Al-Tragma Elgabha Village on the Map (13 Km North of Shandi City). 
was taken as positive according to a study conducted by Ibrahim (9).

\section{Test Establishment and Validation}

At the start of the study, the investigator trained the medical personnel in filling out the questionnaire and performing the LST, with the help of a dermatologist from Shandi Teaching Hospital. The medical team consisted of a medical officer, nurses, lab technicians, and the investigator ( 9 persons). In the field, the team was divided into two groups, one group of four persons under the supervision of the medical officer and the second team consisting of five persons under the supervision of the investigator.

\section{Results}

Most respondents were female $(69.1 \%)$ and $30.9 \%$ were male. The mean age of the respondents was $38.5 \pm 2$. Most of the respondents were housewives (39.2\%), followed by students (22.6\%), farmers (13.6\%), building construction workers (4.9\%), unemployed (15.3\%), office workers (3.2\%), and pensioned persons (1.2\%). The domestic animals and pets found in and around the houses were domestic ruminants (47.2\%), pets (40.1\%), and dogs (1.0\%). Moreover, the rodent burrows were abundant, in and around houses, which were considered as potential reservoir hosts in the area. Most of the respondents slept outdoors (87.8\%) and few of them slept indoors (12.2\%). Additionally, $86.6 \%$ did not use bed nets, $4.4 \%$ used bed nets and $9.0 \%$ were irregular users of bed nets. Moreover, $75.4 \%$ used protective clothes when sleeping and the rest (24.6\%) did not. $91.0 \%$ were not affected by CL before, and only $9.0 \%$ had the disease before.

It was found that $59.5 \%$ of the respondents had CL for more than six years, $35.1 \%$ for $1-3$ years, and $5.4 \%$ for 4- 6 years. Concerning the sites of scars, $73 \%$ of the scars were found in the lower extremities, $16 \%$ in the upper extremities, $8.1 \%$ in all the exposed areas, and $2.9 \%$ in the face. Regarding the number of scars, $89.2 \%$ of them had 1-3 scars, 5.4\% had 4-6 scars, and 5.4\% had more than six scars. In $62.2 \%$ of the respondents, the ulcer healed in $1-3$ months, $10.8 \%$ in $4-6$ months, and $27 \%$ in more than 6 months.

\section{Results of Leishmanin Skin Test (Montenegro Skin Test)}

LST results showed that $70.7 \%$ of respondents were positive while only $29.3 \%$ of the respondents were negative. The diameter of indurations ranged from $5 \mathrm{~mm}$ to $27 \mathrm{~mm}, 48.8 \%$ of the group had an induration size of $5-15 \mathrm{~mm}, 40.5 \%$ had an induration size of $20-25 \mathrm{~mm}$, and only $10.7 \%$ had an induration size of more than $25 \mathrm{~mm}$, (Figure 2; Table 1).

\section{Factors Affecting LST Results}

Among males, $72.2 \%$ (91/126) were positive and 27.8\%
(35/126) were negative. Among females, 70\% (199/284) were positive and $30 \%(85 / 284)$ were negative. There was no significant difference between them $(P=0.658)$ (Table 2).

Table 3 shows the relationship between age groups and leishmanin test results. In the age group 10-20 years, 49\% were positive, in the age group 21-30 years, 69\% were positive, in the age group $31-40$ years, $80 \%$ were positive, and in the age group over 40 years, $82 \%$ were positive. The results showed that there was a significant difference between them $(P=0.005)$.

Concerning the effect of occupation in the result of LST, of the 56 farmers, 46 (82\%) were positive, of the 20 building constructors, 14 (70\%) were positive, of the 13 office workers, 9 (69\%) were positive, of the 93 students, 44 (47\%) were positive, of the 205 housewives, 158 (77\%) were positive, of the 5 pensioned respondents, all 5 (100\%) were positive, and of the 18 unemployed respondents, 14 (77\%) were positive. The results showed that there was a significant difference between them $(P=0.000)$ (Table 4).

As shown in Table 5, concerning the domestic animals and pets found in and around the houses, dogs were found in four houses, and all the people found in these houses were positive, cats and dogs were found in three houses, indicating two positive and one negative results. The results showed that there was a significant difference between them $(P=0.013)$.

Concerning the sleeping habits of the respondents, of the 361 respondents sleeping outdoors, $71.7 \%$ (259/361) were positive and $28.3 \%$ (102/361) were negative. Of the 49 respondents sleeping indoors, 63.3\% (31/49) were positive and $36.7 \%(18 / 49)$ were negative. The results showed no significant difference between them $(P=$ 0.220) (Table 6).

The effect of using bed nets on LST results showed that of the 37 irregular users of bed nets, 67.5\% (25/37) were positive and $32.5 \%(12 / 37)$ were negative. Of the 18 users of bed nets, $78 \%(14 / 18)$ were positive and $22 \%(4 / 18)$

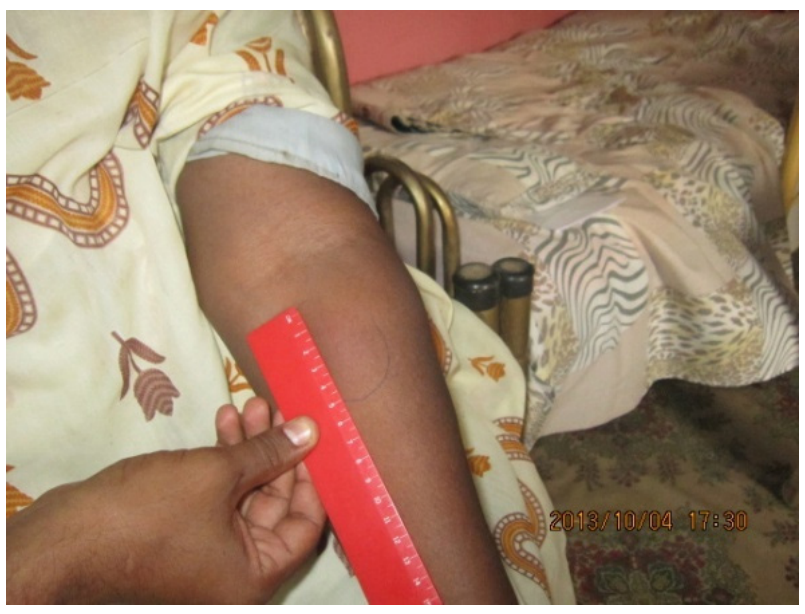

Figure 2. Diameter of Indurations (>25 mm). 
Table 1. The Size of Induration in LST Positive Cases

\begin{tabular}{lcc}
\hline Induration size & No. & Percent \\
\hline$(5-15 \mathrm{~mm})$ & 142 & $48.8 \%$ \\
$(16-26 \mathrm{~mm})$ & 117 & $40.5 \%$ \\
$(27 \geq \mathrm{mm})$ & 31 & $10.7 \%$ \\
Total & 290 & $100.0 \%$ \\
\hline$\chi^{2}=6.134, P=0.040$ & &
\end{tabular}

Table 2. Relationship between LST and Gender

\begin{tabular}{lccc}
\hline & \multicolumn{3}{c}{ Leishmanin Results } \\
\cline { 2 - 4 } & Positive & Negative & Total \\
\hline Males & $91(72.2 \%)$ & $35(27.8 \%)$ & $126(100 \%)$ \\
Females & $199(70 \%)$ & $85(30 \%)$ & $284(100 \%)$ \\
Total & 290 & 120 & 310 \\
\hline
\end{tabular}

$\chi^{2}=0.195, P=0.658$

were negative. Of the 355 non-users of bed nets, $71 \%$ (251/355) were positive and 29\% (104/355) were negative. The results showed no significant difference between them $(P=0.737)$ (Table 7$)$.

The LST results were positive in all the respondents (100\%) who have experienced the disease before (Table $8)$.

\section{Discussion}

The results of this study using LST revealed that the positivity rate of Leishmania major infection was higher in males than in females although the number of females in the study population was higher than males, which could be related to the randomized cluster sampling technique used in this study.

The rearing of domestic animals and the presence of rodents affected the infection rate among the population in the study area. Cats had a major role in the transmission of the disease $(P=0.013)$. Other animals such as ruminants had also a contributory role in transmission. It was observed that rodents, which are considered potential reservoir hosts, were abundant in the study area and burrowed around the houses of residents, not necessarily at the level of the house but at the level of the village at large. This may help in maintaining the transmission cycle. In a study conducted in Palestine, where the causative agent was confirmed to be Leishmania infantum, infected dogs were the primary reservoir hosts in endemic regions, and they were the most significant risk factor predisposing humans to infection (12).

Most of the participants (88\%) used to sleep outdoors, of whom $71.7 \%$ were positive, while only $63.3 \%$ of those sleeping indoors were positive, which may support the claims that outdoor sleeping habits facilitate the transmission of the disease. This finding agreed with the various previous studies done in endemic areas in Sudan (13-16).

LST results revealed that the use of bed nets has no effect on the acquisition of the disease, as most of the participants who did not use bed nets were negative and some who used bed nets were positive. This may be due to the fact that sandflies are active from dusk to dawn and the mosquito bed nets with large mesh sizes are preferred by the community in endemic areas in a hot climate. Nets

Table 3. Comparison of LST Results in Different Age Groups

\begin{tabular}{lcccc}
\hline \multirow{2}{*}{ Age groups } & Total & \multicolumn{2}{c}{ Leishmanin Results } & \multirow{2}{*}{ LST\% (+ve) } \\
\cline { 3 - 4 } & & Positive & Negative & \\
\hline $10-20$ & 91 & 45 & 46 & $49 \%$ \\
$21-30$ & 121 & 84 & 37 & $69 \%$ \\
$31-40$ & 124 & 100 & 24 & $80 \%$ \\
More & 74 & 61 & 13 & $82 \%$ \\
Total & 410 & 290 & 120 & $70.7 \%$ \\
\hline$\chi^{2}=30.788, P=0.005$ & & &
\end{tabular}

Table 4. Comparison of LST Results in Different Occupations

\begin{tabular}{lcccc}
\hline \multirow{2}{*}{ Occupations } & Total & \multicolumn{2}{c}{ Leishmanin } & \multirow{2}{*}{ LST\% (+ve) } \\
\cline { 3 - 4 } & & Positive & Negative & \\
\hline Farmer & 56 & 46 & 10 & $82 \%$ \\
Building constructors & 20 & 14 & 6 & $70 \%$ \\
Office workers & 13 & 9 & 4 & $69 \%$ \\
Students & 93 & 44 & 49 & $47 \%$ \\
Housewives & 205 & 158 & 47 & $77 \%$ \\
Pensioned & 5 & 5 & 0 & $100 \%$ \\
Unemployed & 18 & 14 & 4 & $77 \%$ \\
Total & 410 & 290 & 120 & $70.7 \%$ \\
\hline$\chi^{2}=34.663, P<0.000$ & & & &
\end{tabular}

Table 5. Comparison of LST Results in People Having Different Domestic Animals and Pets

\begin{tabular}{|c|c|c|c|c|c|c|c|c|}
\hline & & \multicolumn{5}{|c|}{ Animals } & \multirow{2}{*}{$N$} & \multirow{2}{*}{ Total } \\
\hline & & C & Do & G, S, Ca & $\mathrm{C}, \mathrm{G}, \mathrm{S}, \mathrm{Ca}$ & $\mathrm{D}, \mathrm{C}$ & & \\
\hline \multirow{2}{*}{ Leishmanin } & + ve & 113 & 4 & 20 & 147 & 2 & 4 & 290 \\
\hline & -ve & 52 & 0 & 20 & 47 & 1 & 0 & 120 \\
\hline \multirow{2}{*}{ Positive $\%$} & & 165 & 4 & 40 & 194 & 3 & 4 & 410 \\
\hline & & $68 \%$ & $100 \%$ & $50 \%$ & $89.7 \%$ & $66.6 \%$ & $0 \%$ & $70 \%$ \\
\hline
\end{tabular}

C: Cat, G: Goats, S: Sheep, Ca: Cattle, D: Dog, N: Not found, T: Total $\chi^{2}=14.423, P=0.013$ 
Table 6. The Comparison LST Results in People with Different Sleeping Habits

\begin{tabular}{|c|c|c|c|c|}
\hline & & \multicolumn{2}{|c|}{ Sleeping Habits } & \multirow{2}{*}{ Total } \\
\hline & & Indoor & Outdoor & \\
\hline \multirow{3}{*}{ LST } & $+v e$ & $31(63.3 \%)$ & $259(71.7 \%)$ & 290 \\
\hline & -ve & $18(36.7 \%)$ & $102(28.3 \%)$ & 120 \\
\hline & Total & 49 & 361 & 410 \\
\hline
\end{tabular}

$\chi^{2}=1.499, P=0.22$

Table 7. Effect of Using Bed Nets on LST Results

\begin{tabular}{lccccc}
\hline & & \multicolumn{3}{c}{ Use of Bed Nets } & \multirow{2}{*}{ Total } \\
\cline { 3 - 5 } & & Rare & Always & Not use & \\
\hline \multirow{2}{*}{ LST } & + ve & $25(67.5 \%)$ & $14(78 \%)$ & $251(71 \%)$ & 290 \\
& -ve & $12(32.5 \%)$ & $4.0(22 \%)$ & $104(29 \%)$ & 120 \\
\multirow{2}{*}{ Total } & & $37(100 \%)$ & $18(100 \%)$ & $355(100 \%)$ & 410 \\
\hline
\end{tabular}

$\chi^{2}=0.611, P=0.737$

Table 8. Relationship Between LST Results and Having a Previous History of the Disease

\begin{tabular}{lcccc}
\hline & & \multicolumn{2}{c}{ Having dieses } & \multirow{2}{*}{ Total } \\
\cline { 3 - 4 } & & Yes & No & \\
\hline \multirow{2}{*}{ LST } & + ve & $37(100 \%)$ & $253(67.8 \%)$ & 290 \\
& -ve & $0(0 \%)$ & $120(32.2 \%)$ & 120 \\
Total & & 37 & 373 & 410 \\
\hline
\end{tabular}

$\chi^{2}=16.829, P=0.000$

with a large mesh size $\left(50\right.$ holes/inch $\left.{ }^{2}\right)$ can prevent the passage of anopheline mosquitoes, but they would allow the passage of the smaller sandflies $(3-5 \mathrm{~mm})$.

The LST results showed that those who have suffered from CL before, as indicated by the presence of scars, were all infected. Scars were reported to be present in different parts of the patients' body, mostly in the lower extremities and to a lesser extent in the upper extremities, and this was because of sandflies lobbing at the lower level. The results also revealed that only one infected child, less than 7 years of age, developed a scar on his face. This finding was contrary to the results reported in Iran by Pourmohammadi et al (17), who indicated that upper extremities are frequently affected sites for the development of scars.

The overall positive rate for LST was 70.7\%, which indicates active transmission of the disease in the study area. Similar results were obtained by Ibrahim (9) in Tuti Island and Al-Tragma Elgabha. The author postulated that the disease is endemic in Al-Tragma Elgabha, and the situation appears to be different from Tuti Island with more clinical cases and features of an outbreak in its peak, either in the ascending or descending phase. It may not be easy to differentiate between these phases without longitudinal follow-up data, which may possibly lead to the initiation of an outbreak in the area (9).

\section{Conclusion}

The overall positive rate for LST was $70.7 \%$ with a higher positivity rate among males compared to females. Furthermore, the exposure duration had no effect on LST results. It seems that the patients acquired long-lasting immunity. LST is still a promising tool for evaluating the epidemiological status in endemic areas, which could help in planning, prevention, and vaccination programs. Effective control can be achieved by the use of bed nets with a small mesh size or insecticide-treated bed nets.

\section{Availability of Data and Materials}

Data are presented within the manuscript and can be provided by the corresponding author upon reasonable request.

\section{Conflict of Interests}

The authors declare that they have no competing interests.

\section{Ethics Issues}

The study protocol was approved by the Research and Ethics Committee of the

Faculty of Medical Laboratory Sciences, University of Gezira and Ministry of Health, River Nile State, Sudan. Written informed consent was obtained from all participants or their guardians/ legal representatives before data and sample collection.

\section{Acknowledgments}

The authors would like to thank all participants recruited for the study. We also thank the medical staff of the Shandi Teaching Hospital for their help during the study.

\section{Authors' Contributions}

AMO, ADA, NMA, and KH conceived and designed the study. $\mathrm{ADA}$ and SBM supervised the study. AMO, NMA, KH, and RSH wrote the initial draft. AMO, ADA, NMA, and KAM analyzed the data and wrote the final draft of the manuscript. AMO, NMA, KH, and RSH contributed to data collection and laboratory investigations. All authors critically read, revised, and approved the final draft of the manuscript submitted to the journal.

\section{Funding}

Not applicable.

\section{References}

1. WHO. (2006). Neglected tropical diseases. Pp: VI. http://apps.who.int/iris/bitstream/handle/10665/69367/ WHO_CDS_NTD_2006.2_eng.pdf; jsessionid=4B1AB4478211658513EE829FA1029A15? sequence $=1$.

2. Alvar J, Vélez ID, Bern C, et al. Leishmaniasis worldwide and global estimates of its incidence. PLoS One. 2012;7(5):e35671. doi:10.1371/journal.pone.0035671

3. Dawit G, Girma Z, Simenew K. A review on biology, epidemiology and public health significance of leishmaniasis. J Bacteriol Parasitol. 2013;4(2):166. doi: 10.4172/2155-9597.1000166.

4. Torres-Guerrero E, Quintanilla-Cedillo MR, RuizEsmenjaud J, Arenas R. Leishmaniasis: a review. F1000Res. 2017;6:750. doi:10.12688/f1000research.11120.1 
5. Karimi T, Sharifi I, Aflatoonian MR, et al. A longlasting emerging epidemic of anthroponotic cutaneous leishmaniasis in southeastern Iran: population movement and peri-urban settlements as a major risk factor. Parasit Vectors. 2021;14(1):122. doi:10.1186/s13071-021-04619-3

6. Sulaiman AA, Elmadhoun WM, Noor SK, et al. An outbreak of cutaneous leishmaniasis among a displaced population in North Sudan: Review of cases. J Family Med Prim Care. 2019;8(2):556-563. doi:10.4103/jfmpc.jfmpc_432_18

7. Okwor I, Uzonna J. Social and economic burden of human leishmaniasis. Am J Trop Med Hyg. 2016;94(3):489-493. doi:10.4269/ajtmh.15-0408

8. Thakur S, Joshi J, Kaur S. Leishmaniasis diagnosis: an update on the use of parasitological, immunological and molecular methods. J Parasit Dis. 2020;44(2):1-20. doi:10.1007/s12639-020-01212-w

9. Naing L, Winn T, Rusli BN. Practical issues in calculating the sample size for prevalence studies. Arch Orofac Sci. 2006;1:9-14.

10. Montenegro J. Cutaneous reaction in leishmaniasis. Arch Derm Syphilol. 1926;13(2):187-194. doi: 10.1001/ archderm.1926.02370140053003.

11. Ibrahim M. The epidemiological components of cutaneous leishmaniasis outbreaks in Tuti Island: a field and simulation approach. WHO, Small Grant Scheme. 2004;195. https:// applications.emro.who.int/dsaf/dsa514.pdf?ua $=1$.

12. Dipineto L, Manna L, Baiano A, et al. Presence of Leishmania infantum in red foxes (Vulpes vulpes) in southern Italy. J Wildl Dis. 2007;43(3):518-520. doi:10.7589/0090-355843.3.518

13. Abdalla NM, Ibrahim ME, El-Hassan AM, et al. Seroepidemiological study on leishmanisis in the Nuba Mountain, Sudan. Turkiye Parazitol Derg. 2000;24(3):228233.

14. Osman AA. Epidemiology of leishmaniasis in south Kordofan region, western Sudan. Res J Med Sci. 2011;5(2):108-11. doi: 10.3923/rjmsci.2011.108.111.

15. El-Hassan AM, Zijlstra EE. Leishmaniasis in Sudan. Cutaneous leishmaniasis. Trans R Soc Trop Med Hyg. 2001;95 Suppl 1:S1-17. doi:10.1016/s0035-9203(01)902160

16. Abdalla NM, Eldosh AA, Abdulgani AM, Yusif BE, Magzoub MM. Typing and characterization of Leishmania sub-clinical isolates from Nuba Mountain, west of Sudan. Infect Genet Evol. 2003;2(4):277.

17. Pourmohammadi B, Motazedian M, Hatam G, Kalantari M, Habibi P, Sarkari B. Comparison of three methods for diagnosis of cutaneous leishmaniasis. Iran J Parasitol. 2010;5(4):1-8. 